\title{
The Interaction of Time with Split-Plot Using Survival Function
}

\author{
Hasan I. Khadher, Kawla M. Sadiq \\ College of Education for Pure Science, Department of Mathematic, University of Mosul, Mosul, Iraq \\ Email:mshasan391@yahoo.com
}

How to cite this paper: Khadher, H.I. and Sadiq, K.M. (2020) The Interaction of Time with Split-Plot Using Survival Function. Open Access Library Journal, 7: e6179. https://doi.org/10.4236/oalib.1106179

Received: February 19, 2020

Accepted: April 23, 2020

Published: April 26, 2020

Copyright $\odot 2020$ by author(s) and Open Access Library Inc.

This work is licensed under the Creative Commons Attribution International License (CC BY 4.0).

http://creativecommons.org/licenses/by/4.0/

\begin{abstract}
It seems that most work has been done for continuous time random variables. Our grouped time models that are used for inference are chosen to relate to these we known continuous time models. We have generalized by the cox (1972) model to include main unit variability to time model.
\end{abstract}

\section{Subject Areas \\ Mathematical Analysis}

\section{Keywords}

Interaction, Split-Plot, Survival Function

\section{Introduction}

\section{Survival Analysis Literature}

Several experimental situations are given rise to analyze time to response on observational units (survival data) using split plot in time models [1] [2]. The general structure of such experiments is that the observation of the time of occurrence of an event (called a death, failure, or response) is of interest. The observational numbers of fishes are grouped in two main units which represented The Acclimation Time (one week and Two weeks), the concentration zinc with three levels (Lo, Me, Hi) and the treatments randomized to each tank. We have two tanks for each level, with daily observation to record results for one week and two weeks. It was designed to study the effect of either one or two weeks acclimation in the test square before introduction to the Zinc.

There were initially two tanks for each of the treatment combinations, the experiment was $(2 \times 3)$ factorial for treatment combinations structure.

The $(2 \times 3)$ treatment combinations were assigned to tanks in a completely 
random design. From this point we used (CRD) to designate this design. The experiment was carried on for (10) days and mortality was observed on daily basis-Three hundred fish were randomized to (12) tanks, (25) fishes to each tank. The $(2 \times 3)$ treatment combinations were assigned 50 that $(2)$ tanks received each treatment.

Table 1 gives a daily mortality for days $1,2,3,4,5,6,7$ where days 8, 9, 10 were each combined gives to (7) [3].

In Table 2 we have interval/ $\hat{q}_{i j k}$

Coming from Risk set TABLE interval $/ \hat{q}_{i j k}$

\section{As in Table 3.}

Assuming that tank effects increase or decrease the survivals, i.e. assuming that there is tank variability involved, since treatment combinations were applied to main units (tanks).

Also assuming that failure time $(T)$ is a discrete random variable since time responses were grouped into intervals $(1,2,3, \cdots, k)$ where $(k=7)$ for the experiment presented. The response for discrete setting would be some function of the number of deaths or the number of survivors.

This will give us a split plot in time where subplot units are time intervals. Failure time variability will arise from the fact that (25) fish were randomly assigned to each tank.

Assuming that conditional on being in the same tank survival times of different fish are independent, then model to be considered is.

$$
\text { Response }=\mu+\propto_{i}+\varepsilon_{i j}+\beta_{k}+(\alpha \beta)_{i k}+\partial_{i j k}
$$

$\mu=$ is an over all mean.

$\propto_{i}=$ is treatment combination ( $\left.i\right)$ effect.

Table 1. Observed number of deaths [9].

\begin{tabular}{|c|c|c|c|c|c|c|c|c|c|c|c|c|}
\hline \multirow{3}{*}{$\begin{array}{c}\text { Acclimation Time: } \\
\text { Zinc Concentration: } \\
\text { Tank: }\end{array}$} & \multirow{2}{*}{\multicolumn{2}{|c|}{$\begin{array}{c}\text { One week } \\
\text { Lo }\end{array}$}} & \multicolumn{10}{|c|}{ Two weeks } \\
\hline & & & \multicolumn{2}{|c|}{ Med } & \multicolumn{2}{|c|}{$\mathrm{Hi}$} & \multicolumn{2}{|c|}{ Lo } & \multicolumn{2}{|c|}{ Med } & \multicolumn{2}{|c|}{$\mathrm{Hi}$} \\
\hline & 1 & 2 & 1 & 2 & 1 & 2 & 1 & 2 & 1 & 2 & 1 & 2 \\
\hline \multicolumn{13}{|l|}{ Day Mortality: } \\
\hline 1 & 0 & 0 & 0 & 0 & 0 & 0 & 0 & 0 & 0 & 0 & 0 & 0 \\
\hline 2 & 1 & 2 & 3 & 0 & 1 & 1 & 0 & 0 & 1 & 0 & 3 & 0 \\
\hline 3 & 5 & 7 & 7 & 10 & 12 & 10 & 9 & 4 & 12 & 9 & 12 & 12 \\
\hline 4 & 7 & 4 & 9 & 7 & 7 & 8 & 4 & 4 & 5 & 3 & 3 & 7 \\
\hline 5 & 1 & 2 & 0 & 5 & 4 & 3 & 0 & 0 & 3 & 2 & 2 & 2 \\
\hline 6 & 0 & 0 & 0 & 1 & 0 & 1 & 0 & 0 & 0 & 0 & 1 & 0 \\
\hline 7 & 0 & 0 & 0 & 0 & 1 & 1 & 0 & 0 & 0 & 0 & 0 & 0 \\
\hline 8 & 0 & 0 & 0 & 1 & 0 & 0 & 0 & 0 & 0 & 0 & 0 & 0 \\
\hline 9 & 0 & 0 & 0 & 0 & 0 & 0 & 0 & 0 & 0 & 0 & 0 & 0 \\
\hline 10 & 0 & 0 & 0 & 0 & 0 & 0 & 0 & 0 & 0 & 0 & 0 & 0 \\
\hline
\end{tabular}


Table 2. Observed value of $q_{i j k \text {. }}$

\begin{tabular}{|c|c|c|c|c|c|c|c|c|c|c|c|c|}
\hline \multirow{3}{*}{$\begin{array}{c}\text { Acclimation Time: } \\
\text { Zinc Concentration: } \\
\text { Tank : }\end{array}$} & \multicolumn{6}{|c|}{ One week } & \multicolumn{6}{|c|}{ Two weeks } \\
\hline & \multicolumn{2}{|c|}{ Lo } & \multicolumn{2}{|c|}{ Med } & \multicolumn{2}{|c|}{$\mathrm{Hi}$} & \multicolumn{2}{|c|}{ Lo } & \multicolumn{2}{|c|}{ Med } & \multicolumn{2}{|c|}{$\mathrm{Hi}$} \\
\hline & 1 & 2 & 1 & 2 & 1 & 2 & 1 & 2 & 1 & 2 & 1 & 2 \\
\hline \multicolumn{13}{|l|}{ Interval/ $\hat{q}_{i j k}:$} \\
\hline 1 & 0.960 & 0.920 & 0.880 & 0.980 & 0.960 & 0.960 & 0.980 & 0.980 & 0.960 & 0.980 & 0.880 & 0.980 \\
\hline 2 & 0.792 & 0.696 & 0.682 & 0.600 & 0.500 & 0.583 & 0.640 & 0.840 & 0.500 & 0.640 & 0.455 & 0.520 \\
\hline 3 & 0.632 & 0.950 & 0.400 & 0.533 & 0.417 & 0.420 & 0.750 & 0.810 & 0.583 & 0.813 & 0.700 & 0.962 \\
\hline 4 & 0.917 & 0.833 & 0.917 & 0.375 & 0.200 & 0.500 & 0.958 & 0.971 & 0.571 & 0.816 & 0.714 & 0.667 \\
\hline 5 & 0.955 & 0950 & 0.917 & 0.667 & 0.500 & 0.667 & 0.958 & 0.971 & 0.875 & 0.955 & 0.800 & 0.875 \\
\hline 6 & 0.955 & 0.950 & 0.917 & 0.500 & 0.500 & 0.500 & 0.958 & 0.971 & 0.875 & 0.955 & 0.875 & 0.875 \\
\hline 7 & 0.955 & 0.950 & 0.917 & 0.500 & 0.500 & 0.500 & 0.958 & 0.971 & 0.875 & 0.955 & 0.875 & 0.875 \\
\hline
\end{tabular}

Table 3. Risk set table.

\begin{tabular}{|c|c|c|c|c|c|c|c|c|c|c|c|c|}
\hline \multirow{3}{*}{$\begin{array}{c}\text { Acclimation Time: } \\
\text { Zinc Concentration: } \\
\text { Tank: }\end{array}$} & \multirow{2}{*}{\multicolumn{2}{|c|}{$\begin{array}{c}\text { One week } \\
\text { Lo }\end{array}$}} & \multicolumn{10}{|c|}{ Two weeks } \\
\hline & & & \multicolumn{2}{|c|}{ Med } & \multicolumn{2}{|c|}{$\mathrm{Hi}$} & \multicolumn{2}{|c|}{ Lo } & \multicolumn{2}{|c|}{ Med } & \multicolumn{2}{|c|}{$\mathrm{Hi}$} \\
\hline & 1 & 2 & 1 & 2 & 1 & 2 & 1 & 2 & 1 & 2 & 1 & 2 \\
\hline \multicolumn{13}{|l|}{ Interval $/ n_{i j k}$} \\
\hline 1 & 25 & 25 & 25 & 25 & 25 & 25 & 25 & 25 & 25 & 25 & 25 & 25 \\
\hline 2 & 24 & 23 & 22 & 22 & 24 & 24 & 25 & 25 & 24 & 25 & 22 & 25 \\
\hline 3 & 19 & 16 & 15 & 15 & 12 & 14 & 16 & 21 & 12 & 16 & 10 & 13 \\
\hline 4 & 12 & 12 & 6 & 8 & 5 & 6 & 12 & 7 & 3 & 13 & 7 & 6 \\
\hline 5 & 11 & 10 & 6 & 3 & 1 & 3 & 12 & 17 & 4 & 11 & 5 & 4 \\
\hline 6 & 11 & 10 & 6 & 2 & 1 & 2 & 12 & 17 & 4 & 11 & 4 & 4 \\
\hline 7 & 11 & 10 & 6 & 2 & 0 & 1 & 12 & 17 & 4 & 11 & 4 & 4 \\
\hline
\end{tabular}

$\varepsilon_{i j}=$ is main unit variability (tank variability) with:

$$
E\left(\varepsilon_{i j}\right)=0, E\left(\varepsilon_{i j} \varepsilon_{i j}\right)=\sigma_{\mathcal{E}}^{2} \text { for } j=j^{\prime}
$$

$B_{k}$ is the subplot treatment or the interval effect [4], $(\propto B)_{i k}$ is the interaction between treatment and time interval. The response of the above will depend on the model assumed for the hazard function for time interval $k$ and Treatment(i) the hazard function $\lambda_{i}\left(t_{k}\right)$ is the conditional probability of failing in an interval given surviving until that interval the choice for response is:

$$
\text { Response }=f\left(\hat{q}_{i j k}\right)
$$

Two possible choices for this function that will be considered are:

$$
\begin{aligned}
& f\left(\hat{q}_{i j k}\right)=\log \left(-\log \hat{q}_{i j k}\right) \\
& \text { and } f\left(\hat{q}_{i j k}\right)=\log \left(\hat{q}_{i j k}\right)
\end{aligned}
$$


Our model for survival analysis is based on using a split plot in time model, and there for we need to consider the related literature as we seen in Table 4 [5] [6]. What we need in the variance component analysis is a method for split-plot models with unequal sub-plot variance. We must mention here that we could not find any work in the literature that has been done for this particular study. However, a list and a presentation of the literature that has been done in both split-plot model and variance component areas separately and combined will be considered some of the listed literature might not be of direct relation to our study and some are related in the sense that they gave us an idea on the approach that we have used for variance component estimation. Then [7]

$$
\begin{gathered}
\log \left(q_{i j k}\right)=\beta^{\prime} Z_{i k}+\mathcal{E}_{i j}^{\prime}+T_{k} \text { where } \beta \in R^{P}, T_{k} \in R \quad \& \\
\log \left(\hat{q}_{i j k}\right)=\log \left(q_{i j k}\right)+\delta_{i j k} \\
\hat{q}_{i j k}=S_{i j k} / \eta_{i j k}
\end{gathered}
$$

$\sigma_{i j k}$ is a random error defined by

$$
\sigma_{i j k}=\log \left(\hat{q}_{i j k}\right)-\log \left(q_{i j k}\right)
$$

It is a proportional hazards model is convenient, e.g. The $\log (-\log )$ model is to be preferred over the large model for the two reasons [8].

1) using the proportional hazards model leads to work with $\log (-\log )$ model specified by the equation.

$$
\log \left(-\log q_{i j k}\right)=\beta^{\prime} X_{i k}+\mathcal{E}_{i j}+\log \int_{t_{k-1}}^{t_{k}} \lambda_{0}(u) \mathrm{d} u
$$

However, using the additive form for the Hazard leads to work with log model specified by the equation.

$$
\log \left(-\log q_{i j k}\right)=\beta^{\prime} X_{i k}\left(t_{k-1}-t_{k}\right)+\mathcal{E}_{i j}\left(t_{k-1}-t_{k}\right)+\left[-\int_{t_{k-1}}^{t_{k}} \lambda_{0}(u) \mathrm{d} u\right]
$$

There for inference with directly related to the parameters of the continuous time interpretation. The $\log (-\log )$ model is to be preferred since $\beta$ is invariant to time grouping.

2) the log model has a restricted range. $\hat{q}_{i j k}$ 's are observed proportions and that $0<\hat{q}_{i j k}<1$ which implies that $\log \left(q_{i j k}\right)<0$.

\section{Main Results}

From analysis not mentioned here we conclude that for $\mathcal{E}_{i j}=0$, the effect of the acclimation time was important in explaining the data. For the first two time intervals there was practically no difference in several rates between acclimation times of one week and two weeks. Fish under two weeks acclimation survived better than these with one week, acclimation time in the sense that the effect became greater with time. This suggests it is better to collect the data (count the number of deaths) after a period of at least three days. There was also an effect due to Zinc 
Table 4. Estimates of binomial variances and values of the response variable.

\begin{tabular}{|c|c|c|c|c|c|c|c|c|}
\hline Accl. & Conc. & Tank & Time & $r_{i j k}$ & $n_{i j k}$ & $S_{i j k}$ & $\sigma_{\delta_{j i k}}^{2}$ & $y_{i j k}$ \\
\hline 1 & 1 & 1 & 1 & 1 & 25 & 24 & 0.747150 & -3.1985 \\
\hline 1 & 1 & 1 & 2 & 5 & 24 & 19 & 0.1728 & -1.4559 \\
\hline 1 & 1 & 1 & 3 & 7 & 19 & 12 & 0.19855 & -0.779 \\
\hline 1 & 1 & 1 & 4 & 1 & 12 & 11 & 0.7531 & -2.4459 \\
\hline 1 & 1 & 1 & 5 & 0 & 11 & 11 & 2.08315 & -3.0782 \\
\hline 1 & 1 & 1 & 6 & 0 & 11 & 11 & 2.08315 & -3.0782 \\
\hline 1 & 1 & 1 & 7 & 0 & 11 & 11 & 2.08315 & -3.0782 \\
\hline 1 & 1 & 2 & 1 & 2 & 25 & 23 & 0.74745 & -2.4843 \\
\hline 1 & 1 & 2 & 2 & 7 & 23 & 16 & 0.1728 & -1.015 \\
\hline 1 & 1 & 2 & 3 & 4 & 16 & 12 & 0.19855 & -1.2459 \\
\hline 1 & 1 & 2 & 4 & 2 & 12 & 10 & 0.7531 & -1.6998 \\
\hline 1 & 1 & 2 & 5 & 0 & 10 & 10 & 2.08315 & -2.9702 \\
\hline 1 & 1 & 2 & 6 & 0 & 10 & 10 & 2.03815 & -2.9702 \\
\hline 1 & 1 & 2 & 7 & 0 & 10 & 10 & 2.08315 & -2.9702 \\
\hline 1 & 2 & 1 & 1 & 3 & 25 & 22 & 1.1878 & -2.057 \\
\hline 1 & 2 & 1 & 2 & 7 & 22 & 15 & 0.12345 & -0.9604 \\
\hline 1 & 2 & 1 & 3 & 9 & 15 & 6 & 0.1333 & -0.0874 \\
\hline 1 & 2 & 1 & 4 & 0 & 6 & 6 & 1.1138 & -2.4459 \\
\hline 1 & 2 & 1 & 5 & 0 & 6 & 6 & 1.5129 & -2.4459 \\
\hline 1 & 2 & 1 & 6 & 0 & 6 & 6 & 2.012 & -2.4459 \\
\hline 1 & 2 & 1 & 7 & 0 & 6 & 6 & 1.5258 & -2.4459 \\
\hline 1 & 2 & 2 & 1 & 0 & 25 & 25 & 1.1878 & -3.9019 \\
\hline 1 & 2 & 2 & 2 & 10 & 25 & 15 & 012345 & -0.6717 \\
\hline 1 & 2 & 2 & 3 & 7 & 15 & 8 & 0.1333 & -0.4633 \\
\hline 1 & 2 & 2 & 4 & 5 & 8 & 3 & 1.1138 & -0.0194 \\
\hline 1 & 2 & 2 & 5 & 1 & 3 & 2 & 1.5129 & -0.904 \\
\hline 1 & 2 & 2 & 6 & 0 & 2 & 2 & 2.012 & -1.2459 \\
\hline 1 & 2 & 2 & 7 & 1 & 2 & 1 & 1.5258 & -0.3665 \\
\hline 1 & 3 & 1 & 1 & 1 & 25 & 24 & 0.9804 & -3.1985 \\
\hline 1 & 3 & 1 & 2 & 12 & 24 & 12 & 0.0946 & -0.3665 \\
\hline 1 & 3 & 1 & 3 & 7 & 12 & 5 & 0.1425 & 0.1339 \\
\hline 1 & 3 & 1 & 4 & 4 & 5 & 1 & 0.3279 & 0.4759 \\
\hline 1 & 3 & 1 & 5 & 0 & 1 & 1 & 1.5479 & -0.3665 \\
\hline 1 & 3 & 1 & 6 & 1 & 1 & 0 & 1.5609 & -0.3665 \\
\hline 1 & 3 & 1 & 7 & 0 & 0 & 0 & 2.0812 & -0.3665 \\
\hline 1 & 3 & 2 & 1 & 1 & 25 & 24 & 0.9804 & -3.1985 \\
\hline
\end{tabular}




\section{Continued}

\begin{tabular}{|c|c|c|c|c|c|c|c|c|}
\hline 1 & 3 & 2 & 2 & 10 & 24 & 14 & 0.0946 & -0.617 \\
\hline 1 & 3 & 2 & 3 & 8 & 14 & 6 & 0.1425 & -0.1669 \\
\hline 1 & 3 & 2 & 4 & 3 & 6 & 3 & 0.3279 & -0.3665 \\
\hline 1 & 3 & 2 & 5 & 1 & 3 & 2 & 1.5479 & -0.904 \\
\hline 1 & 3 & 2 & 6 & 1 & 2 & 1 & 1.5609 & -0.3665 \\
\hline 1 & 3 & 2 & 7 & 0 & 1 & 1 & 2.0812 & -0.3665 \\
\hline-1 & 1 & 1 & 1 & 0 & 25 & 25 & 2.0408 & -3.9019 \\
\hline-1 & 1 & 1 & 2 & 9 & 25 & 16 & 0.1818 & -0.8068 \\
\hline-1 & 1 & 1 & 3 & 4 & 16 & 12 & 0.2516 & -1.2459 \\
\hline-1 & 1 & 1 & 4 & 0 & 12 & 12 & 1.9909 & -3.1487 \\
\hline-1 & 1 & 1 & 5 & 0 & 12 & 12 & 1.9909 & -3.1487 \\
\hline-1 & 1 & 1 & 6 & 0 & 12 & 12 & 1.9909 & -3.1487 \\
\hline-1 & 1 & 1 & 7 & 0 & 12 & 12 & 1.9909 & -3.1487 \\
\hline-1 & 1 & 2 & 1 & 0 & 25 & 25 & 2.0408 & -3.9019 \\
\hline-1 & 1 & 2 & 2 & 4 & 25 & 21 & 0.1818 & -17467 \\
\hline-1 & 1 & 2 & 3 & 4 & 21 & 17 & 0.2516 & -1.5572 \\
\hline-1 & 1 & 2 & 4 & 0 & 17 & 17 & 1.9909 & -3.5258 \\
\hline 1 & 1 & 2 & 5 & 0 & 17 & 17 & 1.9909 & -3.5258 \\
\hline-1 & 1 & 2 & 6 & 0 & 17 & 17 & 1.9909 & -3.5258 \\
\hline-1 & 1 & 2 & 7 & 0 & 17 & 17 & 1.9909 & -3.5258 \\
\hline-1 & 2 & 1 & 1 & 1 & 25 & 24 & 1.5106 & -3.1985 \\
\hline-1 & 2 & 1 & 2 & 12 & 24 & 12 & 0.0999 & -0.3665 \\
\hline-1 & 2 & 1 & 3 & 5 & 12 & 7 & 0.27 & -0.617 \\
\hline-1 & 2 & 1 & 4 & 3 & 7 & 4 & 0.421 & -0.5792 \\
\hline-1 & 2 & 1 & 5 & 0 & 4 & 4 & 2.0231 & -2.0134 \\
\hline-1 & 2 & 1 & 6 & 0 & 4 & 4 & 2.0231 & -2.0134 \\
\hline-1 & 2 & 1 & 7 & 0 & 4 & 4 & 2.0231 & -2.0134 \\
\hline-1 & 2 & 2 & 1 & 0 & 25 & 25 & 1.5106 & -3.9019 \\
\hline-1 & 2 & 2 & 2 & 9 & 25 & 16 & 0.0966 & -0.8068 \\
\hline-1 & 2 & 2 & 3 & 3 & 16 & 13 & 0.27 & -1.5749 \\
\hline-1 & 2 & 2 & 4 & 2 & 13 & 11 & 0.421 & -1.7883 \\
\hline-1 & 2 & 2 & 5 & 0 & 11 & 11 & 2.0231 & -3.0782 \\
\hline-1 & 2 & 2 & 6 & 0 & 11 & 11 & 2.0231 & -3.0782 \\
\hline-1 & 2 & 2 & 7 & 0 & 11 & 11 & 2.0231 & -3.0782 \\
\hline-1 & 3 & 1 & 1 & 3 & 25 & 22 & 1.1872 & -2.057 \\
\hline-1 & 3 & 1 & 2 & 12 & 22 & 10 & 0.0871 & -0.2389 \\
\hline-1 & 3 & 1 & 3 & 3 & 10 & 7 & 0.2436 & -1.0309 \\
\hline
\end{tabular}




\section{Continued}

\begin{tabular}{|c|c|c|c|c|c|c|c|c|}
\hline-1 & 3 & 1 & 4 & 2 & 7 & 5 & 0.5058 & -1.0861 \\
\hline-1 & 3 & 1 & 5 & 1 & 5 & 4 & 1.5052 & -1.4999 \\
\hline-1 & 3 & 1 & 6 & 0 & 4 & 4 & 2.0064 & -2.0134 \\
\hline-1 & 3 & 1 & 7 & 0 & 4 & 4 & 2.0064 & -2.0134 \\
\hline-1 & 3 & 2 & 1 & 0 & 25 & 25 & 1.1877 & -3.9019 \\
\hline-1 & 3 & 2 & 2 & 12 & 25 & 13 & 0.0871 & -0.4248 \\
\hline-1 & 3 & 2 & 3 & 2 & 13 & 6 & 0.2346 & -0.2585 \\
\hline-1 & 3 & 2 & 4 & 2 & 6 & 4 & 0.5058 & -0.904 \\
\hline-1 & 3 & 2 & 5 & 0 & 4 & 4 & 1.5052 & -2.0134 \\
\hline-1 & 3 & 2 & 6 & 0 & 4 & 4 & 2.0064 & -2.0134 \\
\hline-1 & 3 & 2 & 7 & 0 & 4 & 4 & 2.0064 & -2.0134 \\
\hline
\end{tabular}

concentration which indicates that fish survives better with low levels of Zinc concentration than for higher levels.

\section{Conflicts of Interest}

The authors declare no conflicts of interest regarding the publication of this paper.

\section{References}

[1] Lee, E.T. (1992) Statistical Methods for Survival Data Analysis. 2nd Edition, John Wiley \& Sons, Inc., New York.

[2] May, S. and Hostmer, D.W. (2004) An Added Variable Goodness of Fit Test Statistic for the Cox Proportional Hazards Model. In Preparation.

[3] (2001) Agricultural Research, Iraq, Baghdad, Hand Book 2001.

[4] Cox, D.R. and Oakes, S.D. (1984) Analysis of Survival Data. Chapman and Hall, London, New York.

[5] Kleinbaum, D.G. and Klein, M. (2005) Survival Analysis-Aself-Learning Text. 2nd Edition, Springer, New York.

[6] (2014) Survival Analysis, 2nd Edition.

[7] Fieller, N. (2008) Medical Statistics: Survival Data. University of Sheffield, Sheffield.

[8] Kalbfleisch, J.D. and Prentice, R.L. (2002) The Statistical Analysis of Failure Time Data. 2nd Edition, Wiley and Sons, Inc., New York.

[9] Dirienzo, A.G. and Lagakos, S.W. (2004) Hand Book of Statistics Vol. 23. Elsevier B.V., Amsterdam. 